PREPARED FOR THE U.S. DEPARTMENT OF ENERGY, UNDER CONTRACT DE-AC02-76CH03073

PPPL-3602

PPPL-3602

UC-70

Excitation of Accelerating Plasma Waves

by Counter-propagating Laser Beams

by

Gennady Shvets, Nathaniel J. Fisch, and Alexander Pukhov

August 2001

NM|

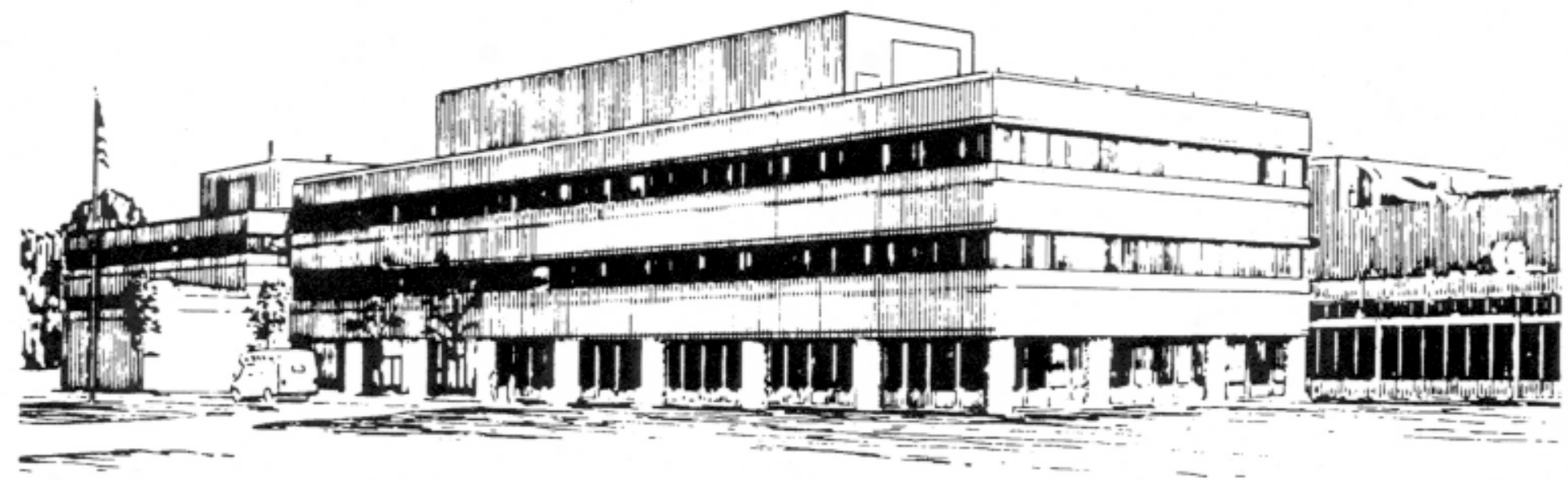

PRINCETON PLASMA PHYSICS LABORATORY PRINCETON UNIVERSITY, PRINCETON, NEW JERSEY 


\section{PPPL Reports Disclaimer}

This report was prepared as an account of work sponsored by an agency of the United States Government. Neither the United States Government nor any agency thereof, nor any of their employees, makes any warranty, express or implied, or assumes any legal liability or responsibility for the accuracy, completeness, or usefulness of any information, apparatus, product, or process disclosed, or represents that its use would not infringe privately owned rights. Reference herein to any specific commercial product, process, or service by trade name, trademark, manufacturer, or otherwise, does not necessarily constitute or imply its endorsement, recommendation, or favoring by the United States Government or any agency thereof. The views and opinions of authors expressed herein do not necessarily state or reflect those of the United States Government or any agency thereof.

\section{Availability}

This report is posted on the U.S. Department of Energy's Princeton Plasma Physics Laboratory Publications and Reports web site in Calendar Year 2001. The home page for PPPL Reports and Publications is: http://www.pppl.gov/pub_report/

DOE and DOE Contractors can obtain copies of this report from:

U.S. Department of Energy

Office of Scientific and Technical Information

DOE Technical Information Services (DTIS)

P.O. Box 62

Oak Ridge, TN 37831

Telephone: (865) 576-8401

Fax: (865) 576-5728

Email: reports@adonis.osti.gov

This report is available to the general public from:

National Technical Information Service

U.S. Department of Commerce

5285 Port Royal Road

Springfield, VA 22161

Telephone: 1-800-553-6847 or

(703) 605-6000

Fax: (703) 321-8547

Internet: http://www.ntis.gov/ordering.htm 


\title{
Excitation of accelerating plasma waves by counter-propagating laser beams
}

\author{
Gennady Shvets and Nathaniel J. Fisch
}

\author{
Princeton Plasma Physics Laboratory ${ }^{1}$ \\ Princeton NJ 08543 \\ Alexander Pukhov \\ Max-Planck-Institut für Quantenoptik, D-85748 Garching, Germany
}

\begin{abstract}
Generation of accelerating plasma waves using two counter-propagating laser beams is considered. Colliding-beam accelerator requires two laser pulses: the long pump and the short timing beam. We emphasize the similarities and differences between the conventional laser wakefield accelerator and the colliding-beam accelerator (CBA). The highly-nonlinear nature of the wake excitation is explained using both nonlinear optics and plasma physics concepts. Two regimes of CBA are considered: (i) the short-pulse regime, where the timing beam is shorter than the plasma period, and (ii) parametric excitation regime, where the timing beam is longer than the plasma period. Possible future experiments are also outlined.
\end{abstract}

\section{INTRODUCTION AND MOTIVATION}

Plasma is an attractive medium for ultra-high gradient particle acceleration because it can sustain a very high electric field, roughly limited by the cold wavebreaking field $E_{\mathrm{WB}}=m c \omega_{p} / e \approx \sqrt{n\left[\mathrm{~cm}^{-3}\right]} \mathrm{V} / \mathrm{cm}$, where $\omega_{p}=\sqrt{4 \pi e^{2} n / m}$ is the plasma frequency and $n$ is the electron density. To accelerate injected particles to velocities close to the speed of light $c$, this electric field has to be in a form of a fast longitudinal plasma wave with phase velocity $v_{\mathrm{ph}} \approx c$. The frequency of the fast plasma wave is $\omega_{p}$, and its wavenumber is $k_{p} \approx \omega_{p} / c$. Excitation of such plasma waves can be accomplished by lasers or fast particle beams [1-3].

Below we review the basics of the linear plasma wave excitation in very general terms, without restricting ourselves to the specifics. Let's assume that plasma electrons are subject to the electric field of the fast plasma wave $\vec{E}$, as well as other nonlinear forces $\vec{F}_{\mathrm{NL}}$, for example, the ponderomotive force of one or more

1) This work was supported by the US DOE Division of High-Energy and Nuclear Physics 
laser pulses. The total current $\vec{J}=\vec{J}_{p}+\overrightarrow{J_{2}}$ which enters Ampere's law $\vec{\nabla} \times \vec{B}=$ $(1 / c) \partial_{t} \vec{E}+(4 \pi / c)\left(\overrightarrow{J_{p}}+\overrightarrow{J_{2}}\right)$ is intentionally split into two components. The first one, $\vec{J}_{p}=-e n \vec{v}_{e}$, where $\vec{v}_{e}$ is the electron fluid velocity, is driven by the electric field $\vec{E}$ and satisfies $\partial_{t} \overrightarrow{J_{p}}=e^{2} n \vec{E}$. The second component $\overrightarrow{J_{2}}$ is driven by the nonlinear ponderomotive force, or could also represent an external current provided by injected electron beam. Taking the time derivative of the Ampere's law yields:

$$
\left(\frac{\partial^{2}}{\partial t^{2}}+\omega_{p 0}^{2}\right) \vec{E}+c^{2} \nabla \times \nabla \times \vec{E}=-4 \pi \frac{\partial \overrightarrow{J_{2}}}{\partial t},
$$

where the $\nabla \times \nabla \times \vec{E}$ term naturally vanishes in $1 \mathrm{D}$. One can say that the science of making a plasma accelerator is about finding the most effective way of producing the appropriate $J_{2 z}(z, t)$. Of course, not every functional form of $J_{2 z}(z, t)$ is useful for relativistic particle acceleration. In the rest of this paper we concentrate on using two counter-propagating laser beams to excite $J_{2 z}(z-c t)$.

\section{COMPARISON OF SINGLE-BEAM AND COLLIDING BEAM ACCELERATORS}

The simplest laser-driven plasma accelerator is the plasma beatwave accelerator [1] (PBWA). It employs a pair of co-propagating laser beams with normalized vector-potentials $\vec{a}_{0,1}=e \vec{A}_{0,1} / m c^{2}$ and frequencies $\omega_{0}$ and $\omega_{1}=\omega_{0}-\omega_{p}$. The nonlinear current $J_{2 z}$ is driven by the ponderomotive force of the resulting electromagnetic beatwave according to $\partial_{t} J_{2 z}=e n \partial_{z}\left(\vec{a}_{0} \cdot \vec{a}_{1}\right)$. If the two laser-beams are detuned by the plasma frequency $\omega_{p}$, plasma wave is resonantly excited.

From Eq. (1), to excite a plasma wave, one needs to deposit momentum into the plasma. The source of this momentum is, of course, the laser. However, since the typical laser frequencies $\omega_{0,1} \gg \omega_{p}$, it is impossible for a laser photon to impart its entire momentum to the plasma. What happens instead is that the frequency of a laser photon is down-shifted by the amount $\omega_{p}$, depositing the remainder momentum and energy into the plasma. In the case of the PBWA, the higherfrequency photons at $\omega_{0}$ are scattered into the lower-frequency photons at $\omega_{1}=$ $\omega_{0}-\omega_{p}$. Schematically, this process is shown in the top Fig. (1). The phasors of the lasers lie on the $\omega^{2}=\omega_{p}^{2}+c^{2} k^{2}$ dispersion curve, and the vector difference of these phasors gives the phasor of the driven plasma wave. The total rate of the momentum transfer to plasma in PBWA is then proportional to the relative momentum transfer per photon $\eta=\omega_{p} / \omega_{0}$, times the rate of scattering which is proportional to the beam intensity. Since the relative amount of down-shifting $\eta \ll 1$, high laser intensities are needed to ensure the high overall rate of the momentum transfer. Note that Fig. 1 (top) is also applicable to the laser wakefield accelerator (LWFA) which employs a single ultra-short $\left(\tau_{L} \approx 2 \omega_{p}^{-1}\right)$ laser pulse. Broad bandwidth of the pulse implies that it contains a continuum of frequency 


\section{Kinematics of Wake Excitation}
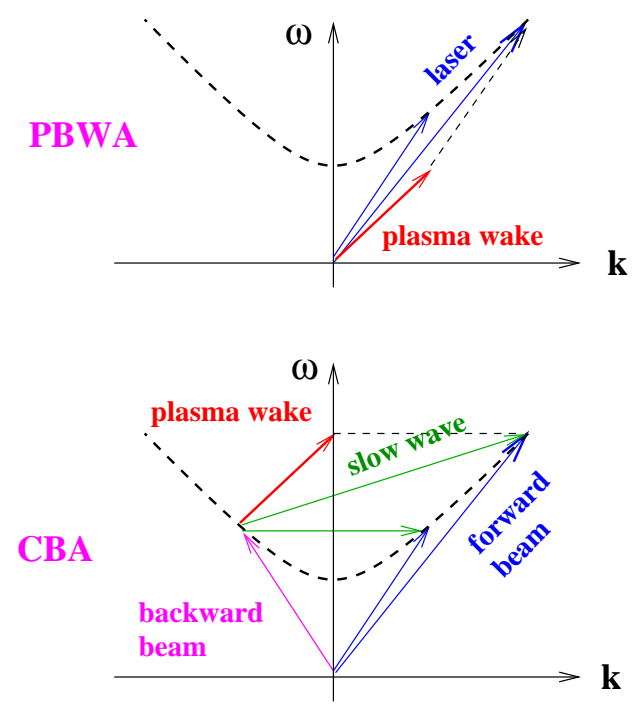

FIGURE 1. PBWA: kinematics of the excitation of the plasma wake by a co-propagating wavepacket consisting of two frequency components differing by $\Delta \omega=\omega_{p}$. Phase velocity of plasma wake $v_{\mathrm{ph}} \approx v g$, where $v_{g}$ is the group velocity of the wavepacket; CBA: same, only using an extra counter-propagating laser beam. Nonlinear beating of two slow waves gives rise to fast plasma wake.

pairs differing from each other by $\omega_{p}$. Because the pulse is short, wake excitation is not resonant, and even larger than in PBWA intensity is needed (typically, close to $10^{18} \mathrm{~W} / \mathrm{cm}^{2}$ to achieve $\left.E / E_{\mathrm{WB}} \sim 0.2\right)$.

In CBA $[4,5]$ we take a very different approach by employing two counterpropagating laser beams with differing frequencies: one short and another long $\left(\tau_{p}=2 L_{p} / c\right.$, where $L_{p}$ is the length of the plasma). When the two beams interact in the plasma, the photons of the higher-frequency beam scatter into the photons of the lower-frequency beam. The crucial difference from the PBWA case is that now approximately twice the total photon momentum is deposited into the plasma: the recoil momentum of scattering a forward moving photon with frequency $\omega_{0}$ into the backward moving photon with frequency $\omega_{2}$ is $\hbar \omega_{0} / c-\left(-\hbar \omega_{2} / c\right) \approx 2 \hbar \omega_{0} / c$. Thus, the laser beams' intensities required to produce a given accelerating field is going to be much smaller for counter-propagating geometry than for the LWFA (or PBWA). More details can be found in Ref. [4].

The bottom drawing in Fig. 1(labeled CBA) illustrates the nonlinear excitation of the fast plasma waves which is significantly more complex than in PBWA (or LWFA). Specifically, we assume that two frequency components, separated by $\omega_{p}$, are propagating in the forward direction. These two frequency components could either belong to two separate and long laser beams (as in PBWA), or two a single ultra-short laser pulse (as in LWFA). In the latter case, a continuum of such fre- 
quency pairs separated by $\omega_{p}$ can be identified. In such pair is shown in Fig. 1. The frequency phasor for the counter-propagating beam is labeled as backward beam. The beating between the different frequency components of the forward beam and the backward beam produce two "slow" plasma waves which are shown as almosthorizontal lines in the drawing. It is the nonlinear mixing of these two slow waves that gives rise to the "fast" plasma wave (labeled as plasma wake). Visually, one can deduce from the drawing that the phase velocity of the fast wave is much larger than that of the slow waves. Mathematically, one can show that the phase velocities of the slow waves roughly scale as $v_{\mathrm{sl}} \approx \omega_{p} / k_{0}$ while the phase velocity of the fast wake is close to the speed of light. In Section II we derive formulas for the fast wake amplitude and demonstrate that, under some circumstances, it can be orders of magnitude larger than the regular wake produced by only the forward propagating pulse(s).

\section{COLLIDING BEAM ACCELERATOR}

The following physical problem was simulated using a one-dimensional particlein-cell (PIC) code VLPL. An ultra-short circularly polarized Gaussian laser pulse with duration $\tau_{L}=1.5 \omega_{p}^{-1}$ and normalized vector potential $a_{0}=0.12$, propagating in the positive $z$ direction, collides in a plasma with a long counter-propagating pulse with $a_{1}=0.05$. Plasma density was chosen such that $\omega_{p} / \omega_{0}=0.05$. The snapshot of the pulse intensity normalized to $2.7 \times 10^{18} \mathrm{~W} / \mathrm{cm}^{2}$ is shown in Fig. 2(a). Two cases, corresponding to the different frequencies of the $\mathrm{PB}, \omega_{1}=1.1 \omega_{0}$ and $\omega_{1}=0.9 \omega_{0}$, were simulated. The resulting plasma wakes are shown in Fig. 2(c) and $(\mathrm{d})$, respectively. For comparison, we also plot the wake produced by a single TB in absence of the counter-propagating pulse in Fig. 2(b). Since the intensity of the short pulse is chosen non-relativistic, the magnitude of the plasma wake left behind the pulse is much smaller than the limiting (wavebreaking) field according to $E / E_{\mathrm{wb}} \sim a_{0}^{2} / 2$, where $E_{\mathrm{wb}}=m c \omega_{p} / e$. The situation changes dramatically when a counter-propagating beam is added. As Figs. 1(c) and (d) indicate, the addition of the pumping beam increases the electric field of the plasma wake by an order of magnitude. To further illustrate this point, we plotted the regular wake [same as shown in Fig. 2(a)] in Figs. 2(c-d) for comparison. Note that the vertical scales of the Figs. 2(c-d) and Fig. 2(b) differ by a factor 20. Plasma wakes produced as a result of the collision between the counter-propagating beams is referred to as the enhanced wake because it is much larger than the regular wake.

This conclusion about the relative magnitudes of the regular and enhanced wakes is only valid for nonrelativistic laser pulses. It turns out that the magnitude of the enhanced wake $E<\left(\omega_{p} / \omega_{0}\right) E_{\mathrm{wb}}$. This limit is set by the maximum velocity of the plasma electrons which cannot significantly exceed the phase velocity of the beatwave between the short and long laser beam, equal to $v_{\mathrm{sl}}=\left(\omega_{0}-\omega_{1}\right) / 2 k_{0}$. Excitation of the fast (accelerating) plasma wake is a strongly nonlinear process, with the "slow" (short-wavelength) plasma waves generated as intermediaries. Wave- 


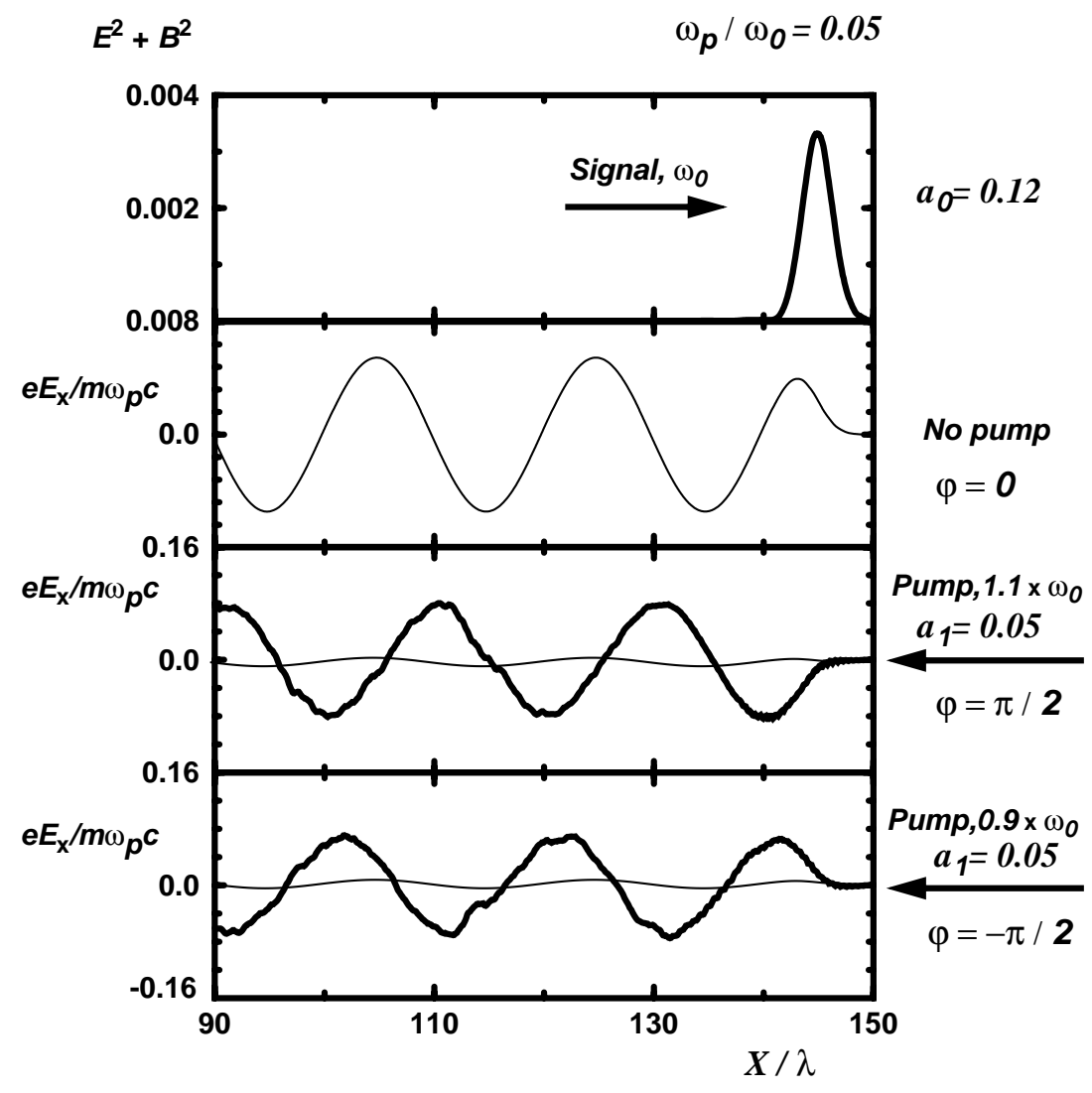

FIGURE 2. Top to bottom: (a) single short laser pulse with $a_{0}=0.12$ and frequency $\omega_{0}$ propagates from left to right; (b) short pulse generates a weak plasma wake $E_{x}$; (c) in the presence of counter-propagating pump with $a_{1}=0.05$ and frequency $\omega_{1}=1.1 \omega_{0}$ the wake is enhanced, and its phase is shifted by $\pi / 2$ with respect to the "regular" wake of (b), which is also shown for comparison; (d) Same as (c), only a down-shifted pump with $\omega_{1}=0.9 \omega_{0}$ is used, and the phase shift is $-\pi / 2$.

breaking of these intermediaries sets limits the excitation of the fast wave. Both the fast and the slow plasma waves are shown in Fig. 1 (bottom).

\section{A Linear regime: four-wave mixing}

The above kinematic illustration in Fig. 1 is, of course, only a cartoon, which does not explain the physical mechanism of the nonlinear mixing between the slow plasma waves. The beating between the slow plasma waves is a novel phenomenon, and we have identified it as a method of driving fast plasma waves. From Eq. (1),

$$
\left(\frac{\partial^{2}}{\partial \zeta^{2}}+\omega_{p}^{2}\right) E_{z}=-4 \pi e \frac{\partial<n v>}{\partial \zeta},
$$


where $\zeta=t-z / c$, and $\langle n v\rangle=\hat{n}_{0} \hat{v}_{1}^{*}+\hat{n}_{1} \hat{v}_{0}^{*}+$ c. c., where $\hat{n}_{0,1}$ and $\hat{v}_{0,1}$ are, correspondingly, fractional density and velocity perturbations in the first and second slow plasma waves. The fast wave, characterized by its amplitude $E_{z}$, is then nonlinearly driven by the RHS of Eq. (2). Equation (2) mathematically expresses the nonlinear mixing between the slow plasma waves schematically shown in Fig. 1. Assuming that pulses 0 and 1 are both flat-tops of duration $\tau_{L}$, the amplitude of the fast wake left behind is

$$
\frac{e E_{z}}{m c \omega_{p}}=\omega_{p} \tau_{L} \frac{\left(\Delta_{0}+\Delta_{1}\right) \omega_{0}^{3}}{\left(\Delta_{0}^{2}-\omega_{p}^{2}\right)\left(\Delta_{1}^{2}-\omega_{p}^{2}\right)}\left|a_{0} a_{1} a_{2}^{2}\right|
$$

where $\Delta_{0}=\omega_{0}-\omega_{2}$ and $\Delta_{1}=\omega_{1}-\omega_{2}$. According to Eq. (3), fast wave generation in the colliding beam accelerator is a four-wave process.

Note that in the particular case of $\Delta_{0}+\Delta_{1}=0$ wakefield vanishes. Since $\omega_{1}=\omega_{0}-\omega_{p}$, this case corresponds to $\omega_{2}=\omega_{0}-0.5 \omega_{p}$. Therefore, the scattering of the photons from beam 0 into beam 2 proceeds at the same rate as the scattering of the beam 2 into beam 1, and the overall momentum deposition into the plasma vanishes. Equation (3) breaks down for $\Delta_{1}^{2}=\omega_{p}^{2}$ and $\Delta_{0}^{2}=\omega_{p}^{2}$. For example, for $\Delta_{1}=0$ and $\Delta_{0}=\omega_{p}$, the wake amplitude is $e E_{z} / m c \omega_{p}=\left(\omega_{0}^{3} \tau_{L}^{2} / 2 \omega_{p}\right) a_{0} a_{1} a_{2}^{2}$.

For a short single-frequency forward-moving pulse, a similar expression for derived in [4] which we present here for completeness:

$$
\frac{e E_{z}}{m c \omega_{p}}=\frac{\pi \Delta \omega}{8 \omega_{0}}\left(4 a_{2} a_{0} \frac{\omega_{0}^{2}}{\omega_{p}^{2}}\right)^{2} \omega_{p}^{2} \tau_{L}^{2} e^{-\omega_{p}^{2} \tau_{L}^{2} / 4}\left[e^{-\left(\omega_{p}-\Delta \omega\right)^{2} \tau_{L}^{2}}+e^{-\left(\omega_{p}+\Delta \omega\right)^{2} \tau_{L}^{2}}+\frac{2}{3} e^{-\Delta \omega^{2} \tau_{L}^{2}}\right]
$$

The most efficient excitation of the accelerating wake requires $\tau_{L} \approx 2.0 \omega_{p}^{-1}$ and $\Delta \omega= \pm 1.1 \omega_{p}$. For these parameters $e E_{z} / m c \omega_{p} \approx 0.6 \omega_{p} / \omega_{0}\left(4 a_{0} a_{2} \omega_{0}^{2} / \omega_{p}^{2}\right)^{2}$. The enhanced wake exceeds the regular wake from forward scattering whenever $a_{2}>$ $\left(\omega_{p} / \omega_{0}\right)^{3 / 2} / 4$. For $n_{0}=10^{18} \mathrm{~cm}^{-3}$, this corresponds to the pump intensity $I_{2}>$ $2 \cdot 10^{14} \mathrm{~W} / \mathrm{cm}^{2}$.

\section{B Nonlinear regime: particle trapping}

The above picture of four-wave process resulting in the excitation of a fast wave is only true when all waves in question are linear. Fast plasma wave always remains linear because its amplitude is below the wavebreaking limit. Slow waves (which interfere to drive the fast wave) break much sooner, their breaking limiting the fast wave amplitude. After wavebreaking, particle motion is determined by ponderomotive beatwave force between counter-propagating beams.

The most interesting and easy-to-understand regime corresponds to the singlefrequency short pulse of duration $\tau_{L}<\pi / \omega_{p}$ which is strong enough to cause wavebreaking. The incidence of wavebreaking is, approximately, determined by 
the ratio of the bounce frequency $\omega_{B}=2 \omega_{0} \sqrt{a_{0} a_{2}}$ and the plasma frequency. In the strongly-nonlinear regime $\omega_{B}^{2} \gg \omega_{p}^{2}$ the amplitude of the plasma wave is estimated [5] as

$$
\frac{e E_{z}}{m c \omega_{p}}=\frac{\left\langle P_{z}\right\rangle}{m c} \sin \omega_{p} \zeta \approx \operatorname{sign}(\Delta \omega)\left(\frac{\omega_{B}}{\omega_{0}}\right) \sin \omega_{p} \zeta
$$

where $\left\langle P_{z}\right\rangle$ is the average momentum transferred to the plasma by the laser pulse. The physics of this momentum transfer can be visualized by plotting the electron phase space at different times: before the arrival of the short pulse, near the maximum of the short pulse, and right after the wavebreaking (Fig. 3). Numerical

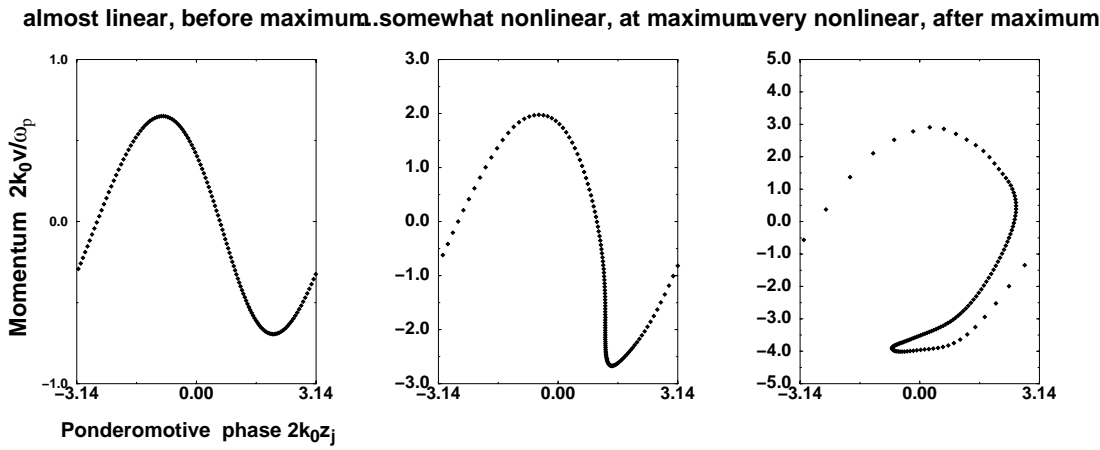

FIGURE 3. Left to right: electron phase space (a) before the arrival of short pulse; (b) near maximum of short pulse; (c) at wavebreaking. Rapid current jolt developing at wavebreaking drives the enhanced wake behind the short pulse.

simulations indicate that the largest momentum gain is achieved for the frequency detuning $\Delta \omega \approx \omega_{B}$ and pulse duration $\tau_{L} \approx 2 / \omega_{B}$. For those parameters, plasma electrons execute about half a bounce in the ponderomotive potential, and leave the ponderomotive bucket with average velocity $v_{z} \approx c \omega_{B} / \omega_{0}$. The nonlinear current $J_{2 z}=-e n v_{z}$ is then inserted into Eq. (1) to yield Eq. (5).

\section{PARAMETRIC EXCITATION OF PLASMA WAVES BY $2 \omega_{P}$ DETUNING}

In the previous section we considered two approaches to excitation of fast plasma waves: one involved two pulses moving in the forward direction and another in the backward direction (beatwave approach), and the other one required a short $\left(\tau_{L} \approx\right.$ $\left.2 / \omega_{p}\right)$ forward-moving pulse and a backward-moving pulse (CBA approach). The beatwave approach is complex for two reasons: (a) three laser pulses are needed, and (b) laser pulses have to be detuned by the plasma frequency. Most laser systems have a fairly small bandwidth (several percent). This forces the plasma density down and reduces the accelerating gradient. CBA can also be challenging because it requires a very short pulse. We have also found from numerical simulations that 
the optimal operation corresponds to $\Delta \omega=(1.5-2.0) \omega_{p}$. This decreases plasma density even further.

All these limitations, and also the simultaneous availability of Nd:Yag $\left(\lambda_{1}=\right.$ $1.06 \mu \mathrm{m})$ and Ti:S $\left(\lambda_{0}=0.8 \mu \mathrm{m}\right)$ laser systems in a number of laboratories compelled us to think of other possible techniques of wake excitation. We suggested a novel scheme [6]: parametric excitation of accelerating plasma waves using counterpropagating laser beams detuned by, approximately, $2 \omega_{p}$. Short-pulse duration no longer is required to be comparable to $\omega_{p}^{-1}$; in fact, it is advantageous to use significantly longer pulses with $\omega_{p} \tau_{L} \approx 25$. From experimental standpoint, this could be a fairly attractive regime: if $\omega_{0}-\omega_{1}=2 \omega_{p}$, then the desired plasma density $n_{p} \approx 2.5 \times 10^{19} \mathrm{~cm}^{-3}$, and the required pulse duration $\tau_{L} \approx 25 \omega_{p}^{-1}$ corresponds to $160 \mathrm{fs}$ (FWHM). Such plasma and laser parameters are achievable, making the practical implementation of our scheme feasible.

That a plasma wave can be driven unstable by the $2 \omega_{p}$ beatwave was originally proposed by Rosenbluth and Liu [7], who calculate the growth rate of a fast plasma wave $\gamma_{R L} \approx \omega_{p} a_{0} a_{1} / 2$ (co-propagating lasers). This instability is high-order, with growth rate scaling as the square of the pump amplitude. Thus, for pump waves of sub-relativistic intensity, i.e. $a_{0}, a_{1} \ll 1$, this decay instability is too slow to be of great practical interest. We realized that (i) the counter-propagating pump geometry results in a growth rate enhanced by the factor $2 \omega_{0}^{2} / \omega_{p}^{2}$, and (ii) fast (accelerating) plasma waves can be produced in the counter-propagating geometry - a fact overlooked in Ref. [7].

Using the equation of motion for the Lagrangian displacement $\zeta=z-z_{0}$,

$$
\ddot{\xi}+\omega_{p}^{2} \xi=i k_{0} c^{2} a_{0} a_{1} e^{i\left[\Delta \omega t-2 k_{0} z\right]}+c . c .,
$$

together with the novel two-wave ansatz for an electron displacement

$$
\xi=A_{f} \sin \left[k_{p} z_{0}-\omega_{p} t+\phi_{f}\right]+A_{s} \sin \left[k_{s} z_{0}-\omega_{p} t+\phi_{s}\right],
$$

where $A_{f}\left(\phi_{f}\right)$ and $A_{s}\left(\phi_{s}\right)$ are the amplitudes (phases) of the fast and slow plasma waves, one can show [6] that both the fast and the slow plasma waves can be driven unstable by a pair of counter-propagating laser beams detuned (approximately) by $2 \omega_{p}$. Of interest to plasma accelerators is, of course, only the fast plasma wave with phase velocity close to the speed of light. We demonstrated that both the fast and the slow waves grow together with the growth rate $\Omega_{i}=\omega_{0}^{2} a_{1} a_{0} / \omega_{p}$ : much faster then for co-propagating lasers. In fact, the presence of the slow wave is very important since it increases the instability growth rate.

The instability mechanism is easy to understand. Fast plasma wave which varies as $\delta n_{f} \sim \cos \omega_{p}(t-z / c)$ modulates the ponderomotive force which oscillates as $f_{z} \sim \cos \left(2 k_{0} z-2 \omega_{p} t\right)$ to resonantly drive the slow wave which varies as $\delta n_{s} \sim$ $\cos \left(2 k_{0}-k_{p}\right) z-\omega_{p} t$. In its turn, the slow wave modulates the ponderomotive force, driving the fast wave and completing the feedback loop of the instability. Instability persists until the wavebreaking of the slow wave. Numerical simulations indicate that the amplitude of the fast wave is limited by $E_{\max }=m c \omega_{p}^{2} / 4 \omega_{0} e$. 
Using a one-dimensional time-averaged particle code, we simulated excitation of the fast and slow plasma waves by a short slightly chirped (under-compressed) pulse with the wavelength $\lambda_{0}=0.8 \mu \mathrm{m}$ which collides with a longer $\lambda_{1}=1 \mu \mathrm{m}$ pulse in a $10^{19} \mathrm{~cm}^{-3}$ plasma. These wavelengths correspond to widely available laser systems (Ti:S and Nd-glass), and the plasma density was chosen to satisfy $\omega_{0}=\omega_{1}+2.35 \omega_{p}$. Other laser parameters are as follows: $a_{0}=0.15 \exp \left[-\zeta^{2} / 2 \tau_{L}^{2}\right]$ with $\tau_{L}=25$ (160 fs FWHM) and $d \delta \omega / d \zeta=-9.5 \times 10^{-3} \omega_{p}$ (3\% bandwidth). The initial fast plasma wave $\tilde{e}_{0}=10^{-3}$ and $a_{1}=0.0165$ have been assumed. There results of the simulation are shown in Fig. 4, where we observe the excitation of both the fast and the slow plasma waves. Despite the small amplitudes of both

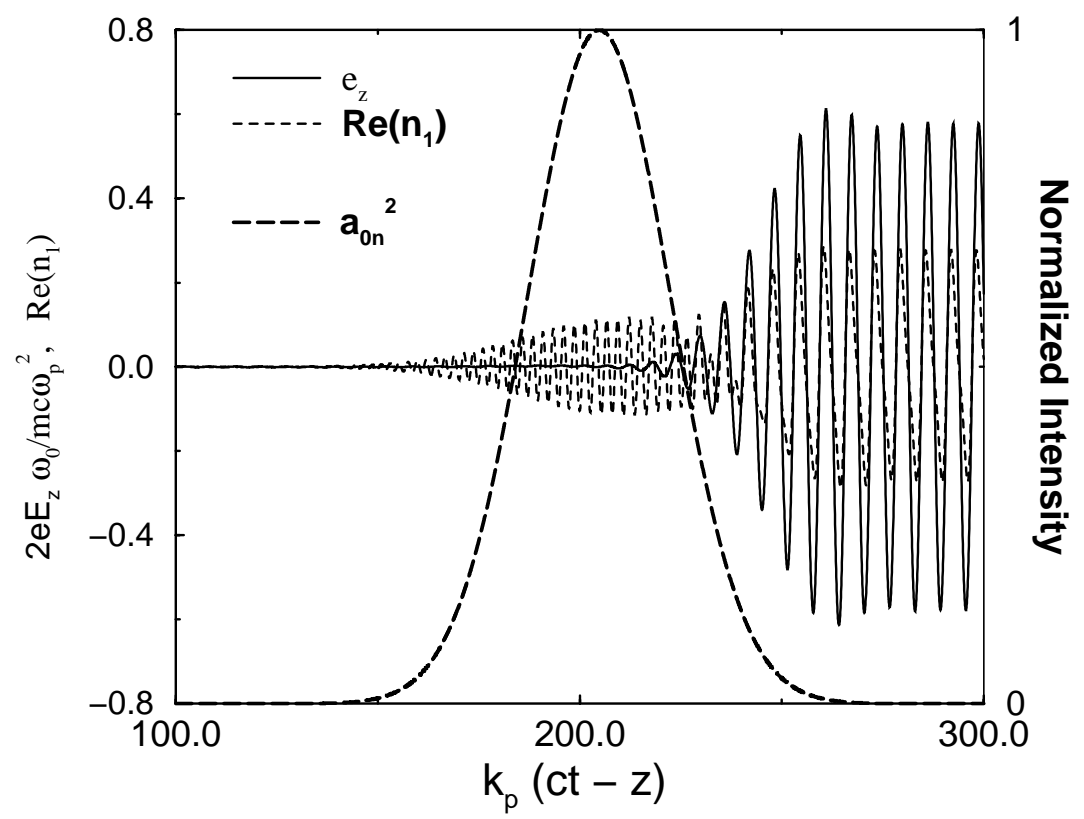

FIGURE 4. Solid line: fast electric field $\tilde{e}_{z}$, long-dashed line: normalized intensity of short pulse $a_{0 n}^{2}$, dashed line: density bunching of the slow plasma wave $\operatorname{Re}\left(\hat{n}_{1}\right)=\left\langle\cos \theta_{j}\right\rangle$. Rapidly-varying part part of $\hat{n}_{1}$ is the driven plasma response inside the laser pulse.

forward and backward pulse, and despite the fact that the duration of the short pulse is too long for the efficient wake generation, we find that a significant fast plasma wave $E_{z}=7 \mathrm{GeV} / \mathrm{m}$ is excited. Parameters used in the simulation are fairly standard for Ti:S and Nd-Glass systems.

\section{UTILITY OF COLLIDING BEAM ACCELERATOR}

One obvious benefit of the counter-propagating geometry is that very large accelerating wakes (of order $10 \mathrm{GeV} / \mathrm{m}$ ) can be produced with moderate-intensity lasers $\left(I \sim 10^{16} \mathrm{~W} / \mathrm{cm}^{2}\right)$. Another, less obvious benefit is the ability to control the phase 
of the accelerating wake. One observes from Fig. 2 that by changing the frequency of the long pulse from $\omega_{1}=1.1 \omega_{0}$ (Fig. 2c) to $\omega_{1}=0.9 \omega_{0}$ (Fig. 2d), the phase of the wake is changed by $\Delta \phi=\pi$. Thus, one can envision a "plasma linac" which consists of independently phase-controlled acceleration sections, separated by drift spaces.

Numerical implementation of the "plasma linac" concept is shown in Fig. 5. Collision of a short "timing beam" (TB) of duration $\tau_{L}=\omega_{p}^{-1}$ and normalized vector potential $a_{0}=0.08$ with a long "pumping beam" (PB) $a_{1}=0.012$ is modeled using a 1D version of the Particle-in-Cell (PIC) simulation code VLPL. Fig. 5(a) illustrates the temporal profile of the PB, which moves to the left; Figs. 5(b,c) are the snapshots of the generated plasma wake and the phase space of accelerated electrons, which are continuously injected with initial energy $10 \mathrm{MeV}$ electrons; Fig. 5(d) shows the evolution of the TB as it moves through the plasma. To show how one can control the phase and the magnitude of the resulting plasma wake, we split the PB into two sections: the leading section of duration $\Delta t_{1}=500 \times 2 \pi / \omega_{0}$, where $\Delta \omega=-1.7 \omega_{p}$, and the trailing section $\Delta t_{3}=250 \times 2 \pi / \omega_{0}$, where $\Delta \omega=1.7 \omega_{p}$. These two pump beam sections are separated by the middle section of duration $\Delta t_{2}=\Delta t_{3}$, where the pump is switched off.

As Figs. 5(a,b) show, the three pump sections map into three spatial acceleration regions, which are different from each other in TB dynamics, magnitude, and phase of the plasma wake. In the leading region the pump beam has higher frequency and energy flows into the TB, amplifying it. A strong plasma wake with the peak accelerating gradient of $8 \mathrm{GeV} / \mathrm{m}$ is induced. The middle region is void of the pump. Here the TB interacts with the plasma through the usual LWFA mechanism only, producing a weak, $<1 \mathrm{GeV} / \mathrm{m}$, accelerating wake. In this region the energy of the injected electrons does not significantly change, as seen from Fig. 5(d). When the trailing (low-frequency) part of the pump collides with the TB, the energy flows from the TB into the PB, Fig. 5(c). Again, a strong plasma wake is induced, Fig. 5(b). This wake, however, is shifted in phase by $\Delta \phi=\pi$ with respect to the leading region. As a result, electrons which gained energy in the leading region are decelerated in the trailing region, Fig. 5(d). This shows that both amplitude and phase of the enhanced plasma wake can be controlled by shaping the long low-intensity pump beam.

Plasma linac can be used to prevent phase slippage between ultra-relativistic particles and the wake which has the phase velocity $v_{\mathrm{ph}} / c \approx 1-\omega_{p}^{2} / 2 \omega_{0}^{2}$. Since particles are moving slightly faster than the wake crests, they eventually outrun the accelerating phase and move into the decelerating phase of the wake (Fig. (6), left). This occurs after one dephasing length $L_{d}=\lambda_{p}^{3} / \lambda_{0}^{2}$. After that, acceleration has to be terminated by terminating the plasma. The next acceleration stage needs to be in phase with the previous one, presenting a serious technical challenge.

In a colliding beam plasma linac shown in Fig. (5) dephasing can be circumvented by taking the length of the leading pump section equal to $2 L_{d}$. Particle phase dynamics is shown in Fig. (6), right. After advancing in phase by $\Delta \phi=\pi$, electron finds itself in the gap between accelerating sections. Accelerating field in the gap is 

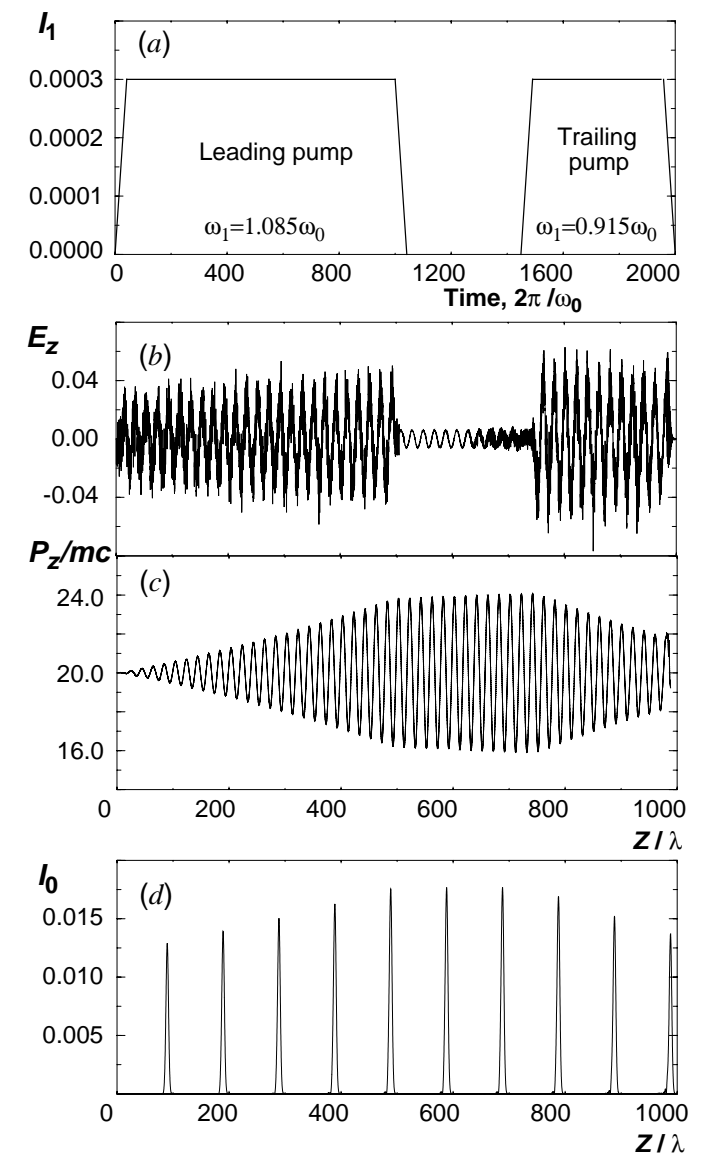

FIGURE 5. Collision between a short timing beam $\left(a_{0}=0.08, \tau_{L}=\omega_{p}^{-1}\right)$ and an intermittent pump $\left(a_{1}=0.012\right)$ in $n_{0}=2.5 \times 10^{18} \mathrm{~cm}^{-3}$ plasma $\left(\omega_{0} / \omega_{p}=20\right) .10 \mathrm{MeV}$ electrons are continuously injected into the plasma. (a) Time-dependence of the pumping beam intensity $I_{1}=a_{1}^{2}$; (b) longitudinal electric field $e E_{z} / m c \omega_{0}$; (c) propagation of the TB through the plasma, $I_{0}=a_{0}^{2}$; (d) phase space of injected electrons.

very small because there is no enhanced wake there. After the gap, electron enters the second accelerating section, where the phase differs from the first section by $\pi$. Therefore, electron is in the accelerating phase again. This sequence can be repeated indefinitely, ensuring that electron is never decelerated.

\section{FUTURE WORK}

An important unresolved problem is generation of accelerating plasma waves using the CBA technique in a plasma channel. Plasma channels are important for guiding both long and short laser beams. Moreover, transversely inhomogeneous plasma may impart an unusual structure of the accelerating field with a local minimum on axis. This may result in advantageous transverse focusing properties of the wake, especially in the context of the colliding-beam injector [5]. 

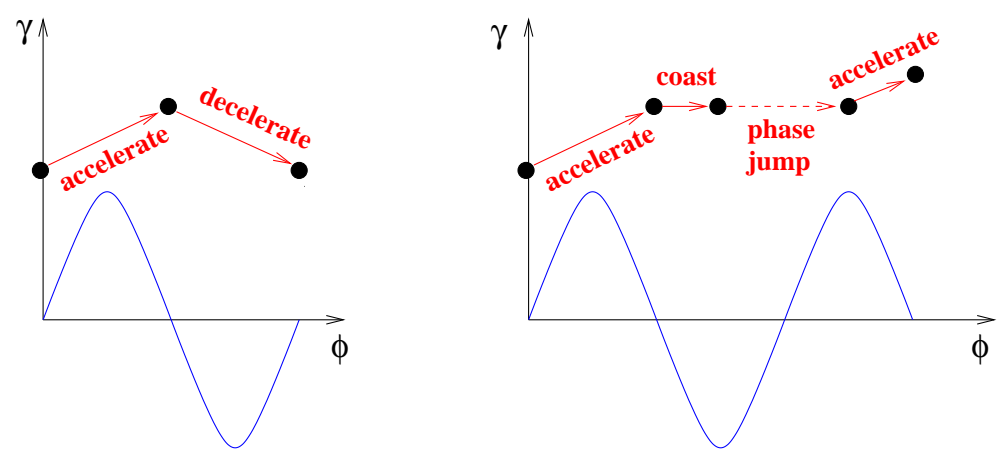

FIGURE 6. Schematic of the phase slippage of electron with respect to the wake in a standard wakefield accelerator (left) and in a "plasma linac" (right).

This work was supported by the DOE Division of High Energy Physics and the Presidential Early Career Award for Scientists and Engineers.

\section{REFERENCES}

1. T. Tajima and J. M. Dawson, Phys. Rev. Lett. 43, 267 (1979).

2. P. Chen, J. M. Dawson, R. W. Huff, and T. Katsouleas, Phys. Rev. Lett. 54, 693 (1985).

3. E. Esarey, P. Sprangle, J. Krall, and A. Ting, IEEE Trans. Plasma Science 24, 252 (1996), and references therein.

4. G. Shvets, N. J. Fisch, A. Pukhov, and J. Meyer-ter-Vehn, Phys. Rev. E 60, 2218 (1999).

5. G. Shvets, N. J. Fisch, and A. Pukhov, IEEE Trans. Plasma Science, 28, 1194 (2000).

6. G. Shvets and N. J. Fisch, Phys. Rev. Lett. 86, 3328 (2001).

7. M. N. Rosenbluth, C. S. Liu, Phys. Rev. Lett. 29, 701 (1972). 


\section{External Distribution}

Plasma Research Laboratory, Australian National University, Australia

Professor I.R. J ones, Flinders University, Australia

Professor J oão Canalle, Instituto de Fisica DEQ/IF - UERJ , Brazil

Mr. Gerson O. Ludwig, Instituto Nacional de Pesquisas, Brazil

Dr. P.H. Sakanaka, Instituto Fisica, Brazil

The Librarian, Culham Laboratory, England

Library, R61, Rutherford Appleton Laboratory, England

Mrs. S.A. Hutchinson, JET Library, England

Professor M.N. Bussac, Ecole Polytechnique, France

Librarian, Max-Planck-Institut für Plasmaphysik, Germany

J olan Moldvai, Reports Library, MTA KFKI-ATKI, Hungary

Dr. P. Kaw, Institute for Plasma Research, India

Ms. P.J . Pathak, Librarian, Insitute for Plasma Research, India

Ms. Clelia De Palo, Associazione EURATOM-ENEA, I taly

Dr. G. Grosso, Instituto di Fisica del Plasma, Italy

Librarian, Naka Fusion Research Establishment, J AERI, J apan

Library, Plasma Physics Laboratory, Kyoto University, J apan

Research Information Center, National Institute for Fusion Science, J apan

Dr. O. Mitarai, Kyushu Tokai University, J apan

Library, Academia Sinica, Institute of Plasma Physics, People's Republic of China

Shih-Tung Tsai, Institute of Physics, Chinese Academy of Sciences, People's Republic of China

Dr. S. Mirnov, TRINITI, Troitsk, Russian Federation, Russia

Dr. V.S. Strelkov, Kurchatov Institute, Russian Federation, Russia

Professor Peter Lukac, Katedra Fyziky Plazmy MFF UK, Mlynska dolina F-2, Komenskeho Univerzita, SK-842 15 Bratislava, Slovakia

Dr. G.S. Lee, Korea Basic Science Institute, South Korea

Mr. Dennis Bruggink, Fusion Library, University of Wisconsin, USA

Institute for Plasma Research, University of Maryland, USA

Librarian, Fusion Energy Division, Oak Ridge National Laboratory, USA

Librarian, Institute of Fusion Studies, University of Texas, USA

Librarian, Magnetic Fusion Program, Lawrence Livermore National Laboratory, USA

Library, General Atomics, USA

Plasma Physics Group, Fusion Energy Research Program, University of California at San Diego, USA

Plasma Physics Library, Columbia University, USA

Alkesh Punjabi, Center for Fusion Research and Training, Hampton University, USA

Dr. W.M. Stacey, Fusion Research Center, Georgia Institute of Technology, USA

Dr. J ohn Willis, U.S. Department of Energy, Office of Fusion Energy Sciences, USA

Mr. Paul H. Wright, Indianapolis, Indiana, USA 
The Princeton Plasma Physics Laboratory is operated by Princeton University under contract with the U.S. Department of Energy.

\author{
Information Services \\ Princeton Plasma Physics Laboratory \\ P.O. Box 451 \\ Princeton, NJ 08543
}

Phone: 609-243-2750

Fax: 609-243-2751

e-mail: pppl_info@pppl.gov

Internet Address: http://www.pppl.gov 